\title{
Efficiency of ZigBee Wireless Communication in Aircraft Environment
}

\author{
${ }^{1}$ Ahmad Ghulam Baksh, ${ }^{2}$ Dr. Al Savvaris, ${ }^{3}$ Prof. Antonios Tsourdos
}

\begin{abstract}
The use of ZigBee wireless sensor network has increased, covering various application areas. The different features of ZigBee like self-healing, robustness, support for mesh topology has made it as an attractive option for use in industrial applications. In this paper the efficiency of using the ZigBee communications in an aircraft environment is analyzed. More specifically, the efficiency of the wireless communications between the sensors present in the aircraft wings with the controllers located in the cabin is analyzed. An earlier design of the aircraft wiring model is utilized to simulate the radiowave propagation. CST (Computer Simulation Technology) software is used to produce the simulations in this work. The paper concludes by providing the impact of the wing shape and the wing inner structure on the radiowave propagation present in the aircraft wing.
\end{abstract}

Keywords - ZigBee, wireless sensor network, radio wave propagation, Wing shape, wing inner structure

\section{Introduction}

Aircraft maintenance is an important and also challenging task in which installation and the maintenance of the wired communication system is highly challenging issue. These traditional wired communication systems are not only difficult to maintain but are also costly in nature. Today with the increase in competition level, the airline operators are pressured to minimize the cost and to improve the profitability of running the fleet. This pushed the aircraft manufactures to continuously look for techniques and technologies through which the aircraft can be designed to be lighter and which can help in saving the fuel consumption of the aircraft and reduce maintenance costs. Since aircraft wiring is

\footnotetext{
${ }^{1}$ Ahmad Ghulam Baksh

Cranfield University, Aerospace Department SATM United Kingdom

${ }^{2} \mathrm{Al}$ Savvaris

Cranfield University, Aerospace Department SATM

United Kingdom

${ }^{3}$ Antonios Tsourdos

Cranfield University, Aerospace Department SATM

United Kingdom
}

considered as one part of the aircraft system that requires high cost for the installation and maintenance [1], this paper mainly concentrates in minimizing the cost of communication so that the operation cost of aircrafts can be minimized. The idea behind minimizing the communication cost is replacing the wired loom with the wireless connections [2].

The developments in the wireless technology expanded the deployment of wireless communication systems to wide range of application areas. This research concentrates on one of the commonly used wireless communication protocols, namely, ZigBee. ZigBee is based on IEEE 802.15.4 standard. It is a short-range, low data rate and low power protocol. These characteristics of the ZigBee technology has made it to be used in different application areas like industries, construction, medicine, transportation and several others [3]. Examples of other areas in which the ZigBee wireless network is applied, is in the case of a rescue system used in the coal mines [4]; wireless network used for monitoring the humidity levels and the temperature levels of the soil [5]. ZigBee wireless network is also being used in the maintenance of the civilian flight systems [6]. The current work attempts to apply ZigBee wireless communications in aircraft wing and is mainly focused on using the wireless sensors for transmitting the data that is originated from the engine present in the wing to the main controller present in the cabin of the aircraft. The low cost designs and low power consumption make the ZigBee wireless communications an attractive solution for use in aircraft environments [2].

\section{Structure and topologies of ZigBee technology}

\section{A. ZigBee}

ZigBee technologies offer lower power consumption and are relatively less costly when compared to Bluetooth devices. The specifications of ZigBee are based on IEEE 802.15.4 standard. The ZigBee radio works in the similar ISM band as that of the Bluetooth and has the capacity to connect nearly 255 devices in each network. Through these ZigBee specifications, a data rate of nearly $255 \mathrm{Kbps}$ is 
supported with transmission range of nearly 30 meters [7]. Even though ZigBee offers slower data rates; the power consumption of these devices is lower, while providing longer transmission range. The main aim of the ZigBee standard is to offer a solution for devices that are deployed and expected to operate for several months or even a year without the need for recharging. For example, in the case of wireless sensor network deployed in an industrial plant.

In a ZigBee network three types of devices exists, namely: network coordinator, router and the end devices. The network coordinator acts as the main controller of the network through which the complete ZigBee network can be effectively monitored. The network coordinator helps in both starting the communication and also in ending it [8]. The other type of device is the router which is a FFD (Full Function Device). The main function of the router is to offer synchronization of the services for the network coordinators and also for the end devices. These routers also help in dynamically routing the information and in offering the backup routes in the case when there is any failure in any of the ZigBee devices [18]. With the help of these routers, the network coverage area can be increased in the ZigBee networks. Furthermore, these routers can be used in the devices which act as the edges of the network.

The third type of device which can be supported by these ZigBee networks is the end device. This end device will not have any functionality to route the information, it can easily receive the information and can transmit it [9]. These end devices come under the RFDs (Reduce Function Devices) and hence they are not required to deal with the large amount of data. The end devices have simpler structure associated with a 64-bit address value [19]. Due to minimum requirements with respect to memory and the power, these end devices can also have only 16-bit address.

\section{B. Topology of the ZigBee Networks}

The IEEE 802.15.4 ZigBee standard mainly exists in two different topologies. These topologies will be utilized during the design of the ZigBee networks.

Star Topology: The star topology is considered to be a very simple topology and is also treated as the least utilized topology in the ZigBee standard. In this star topology, one network coordinator is present along with the several RFDs [9]. The transmission of the data can take place from one RFD to another RFD only via the network coordinator. Hence the coordinator will be acting as the router. Even though this star topology can be constructed very easily, there is the limitation of the inability to establish the route when the connection between the RFD and the network coordinator is removed. The presence of the network coordinator results in the single point failures in this star topology [10].

Mesh Topology: The mesh topology on the other hand consists of the network coordinator and this coordinator will have connections with the RFDs and also the FFDs [11]. In general there will be a connection between the router and the FFDs so that the routing can be performed between the nearest neighboring nodes. As there is direct communication between the routers and the FFDs, the rules of transmission and receiving will be very flexible in this mesh topology. The use of the mesh topology improves the reliability of the network and moreover improves the packet propagation. In case of any failure in the device, the router can reroute the data through the other FFDs [16]. The use of the mesh topology also supports the multi-hop communication.

The use of this mesh topology can increase the range of the network coverage, as this topology has large number of routers. In the mesh topology the occurrence of dead zones and weak signals can be easily resolved when the number of FFDs is increased. In case of any device failure, the rerouting of the data will be made using another FFD [12]. This topology can be self-configured and also can be selforganized through which the network reliability and the robustness can be increased.

\section{WSN in the Aircraft Environment}

The improvements in performance of wireless sensor networks enabled their use in several sensitive application areas such as habitat monitoring; or object tracking in military applications. Therefore, the potential of exploiting them in an aircraft environment was a rational step in moving forward. The utilization of the wireless sensor networks inside the aircraft can offer several advantages such as reducing the wiring looms weight, making the aircraft design simpler and hence overall reducing the aircraft manufacturing costs, weight and complexity [13]. However, fitting wireless sensor networks inside an airplane is not a straight forward matter. Certain challenges must be taken into consideration.

These challenges include the interference in the aircraft structures; interference generated by other sensors and systems onboard the aircraft; security of data transmissions and the risks associated with someone hacking into the system; and provision of 
the non-failure work environment in which other wireless systems will be working [15, 17]. This paper will investigate one of those challenges, namely, the practicability of using ZigBee wireless sensor devices and the radio-propagation in the $2.4 \mathrm{GHz}$ frequency band experienced inside an aircraft wing inner structure. This paper also helps in identifying that IEEE 802.15.4 standard provides proper resistance for the interference which arises in the airplane environment where $2.4 \mathrm{GHz}$ frequency band is used. Figure 1, illustrates the sensor network for aircraft health monitoring. This configuration is similar to that envisaged in the future to be used for aircraft Structural Health Monitoring (SHM), where data are collected in real-time.

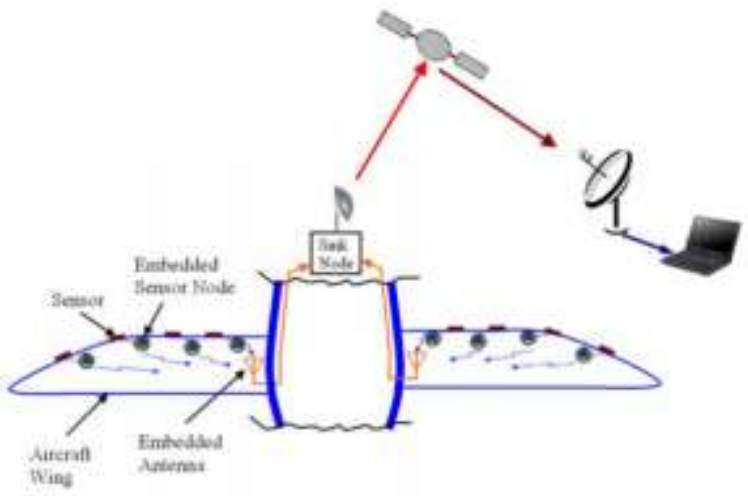

Figure 1. Configuration of WSN for aircraft SHM

In above schematic the wireless devices are considered to operate in the frequency band of 2.4 GHz. Checking of the wireless nodes will also be done in the same operating frequency band of $2.4 \mathrm{GHz}$, in order to reduce the packet loss which may arise between the two nodes in the network [14].

\section{Design of Wing Model}

To analyse the radiowave propagation inside the aircraft wing, three wing models were designed. The CAD software that was used to design these three wing models was Catia. As the simulation software considers the over wall gauges or over wall surfaces, there will not be any thickness for the external wing and the internal surfaces. The three wing models which were created in this work that are NACA64A410 model for inner structure of the wing, empty NACA64A410 model for the wing and empty Prismatic model of the wing. The different dimensions of the aeroplane wing are considered. The size of the wing was selected based on that of a single aisle passenger aeroplane, such as the Airbus A320 or Being 737. The first two wing models are based on the NACA64A410 aerofoil. The third wing uses a Prismatic model; the thickness of the wing of this third model is equal to $598 \mathrm{~mm}$ (Maximum thickness) of the wing route.

\section{v. Analysis of Simulation Data}

This section presents the analysis of the simulation results obtained from the wing models in CST software. The analysis of the different antenna positions on the wing indicates that antenna position has a great impact on the radiowave propagation. When comparing the realistic wing model and the prismatic wing model, the results shows the difference with respect to coupling between the transceivers at different positions of the antenna. Based on the theory, the signal strength between the antennas should be high when they are closer and should be less when they are distant apart. From the simulation the coupling that is present between the transceivers is high when they are far apart. The main reason for this effect is due to the high reflections inside the wing cavity and the high resonance effect that is caused from the ribs and the shape of the wing.

In the simulation the lowest value of coupling $-73 \mathrm{~dB}$ is obtained when the second transceiver is placed at $1 / 4$ length of the wing in the empty wing model at the frequency of $2.465 \mathrm{GHz}$. In case of prismatic model the lowest coupling value was obtained when the second transceiver is placed at $1 / 2$ length of the wing and also at $3 / 4$ length of the wing. In case of the first ZigBee channel the best coupling value is $-30 \mathrm{~dB}$ and the worst value is $-63 \mathrm{~dB}$, which is obtained between the antennas that are located at $3 / 4$ of the wing length in the prismatic wing model. This is because of the lower level of resonance and also less level of reflection when compared with the high complex shapes. The comparison of the S21 parameter values for different position of the antenna in different wing model is shown in the Table 1 below:

TABLE1. COMPARISON OF $S_{21}$ PARAMETER VALUES FOR DIFFERENT POSITION OF ANTENNA IN DIFFERENT WING

\begin{tabular}{|c|c|c|}
\hline Wing Model & 2"it Tranescoiver Position & $S_{21}$ Value [dB] \\
\hline \multirow{4}{*}{ realistic NACA64A410 } & $1 / 4$ wing length & $\sin 289.9$ \\
\hline & $1 / 2$ wine leagth & $-57,1277$ \\
\hline & $3 / 4$ wing leangth & -216.3342 \\
\hline & wing tip & $-11.6641)$ \\
\hline \multirow{4}{*}{ amply NACA64A410 } & $1 / 4$ wing length & $-47,0254$ \\
\hline & 1/2 wing length & -123665 \\
\hline & 3/4 wing length & - 6 6) 0134 \\
\hline & wing tup & -37.1754 \\
\hline \multirow{4}{*}{ privan modr| } & $1 / 4$ wing length & -629906 \\
\hline & 1/2 wing lemgth & $-17,+6664$ \\
\hline & 3/4 wing length & $-311,7560$ \\
\hline & wing tip & $-11 .+5 \times 4$ \\
\hline
\end{tabular}


Proc. of the Fourth Intl. Conf. Advances in Computing, Communication and Information Technology- CCIT 2016

Copyright $\odot$ Institute of Research Engineers and Doctors, USA .All rights reserved.

ISBN: 978-1-63248-092-7 doi: 10.15224/ 978-1-63248-092-7-70

\section{A. Influence of the wing shape and inner structure}

The initial analysis that is made regarding the coupling of two antennas with respect to wing length is the identification of influence made by shape of the wing on the performance. Figures 2 to 5, below, show the comparison of the value of S21 parameter with respect to different models of the wing, namely: empty model, prismatic model and realistic model when the antenna positions are changed based on wing length.

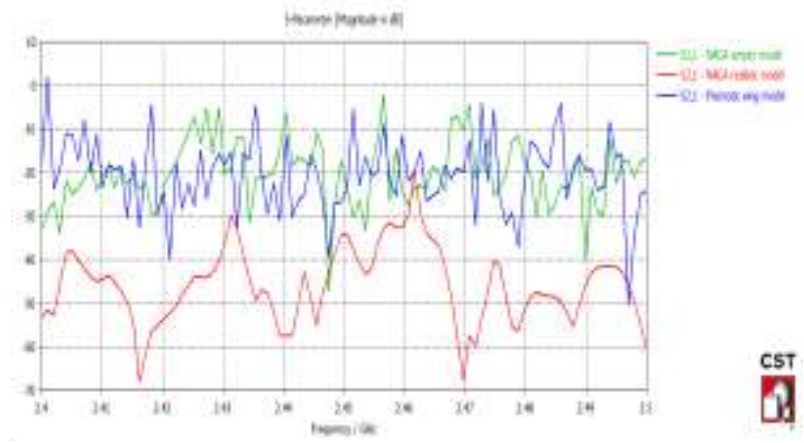

Figure 2. Position: 1/4 length of the wing

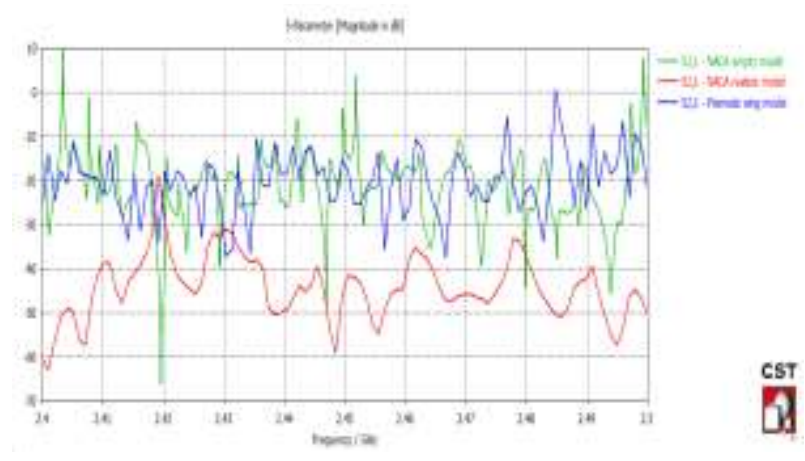

Figure 3. Position: $1 / 2$ length of the wing

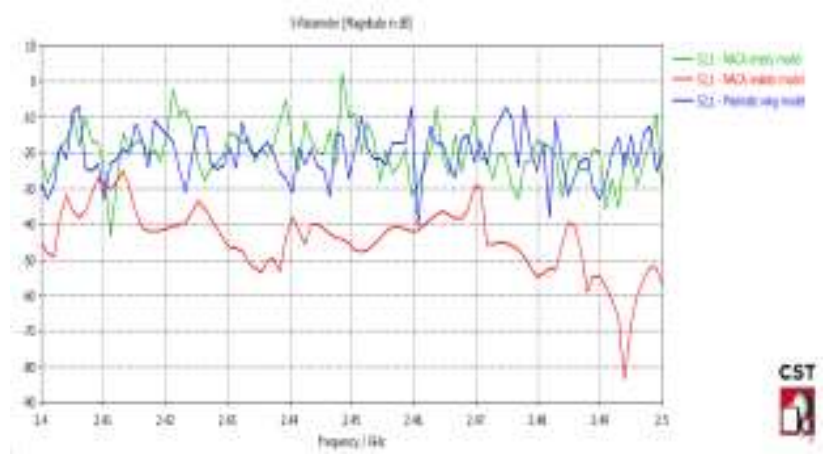

Figure 4. Position: $3 / 4$ length of the wing

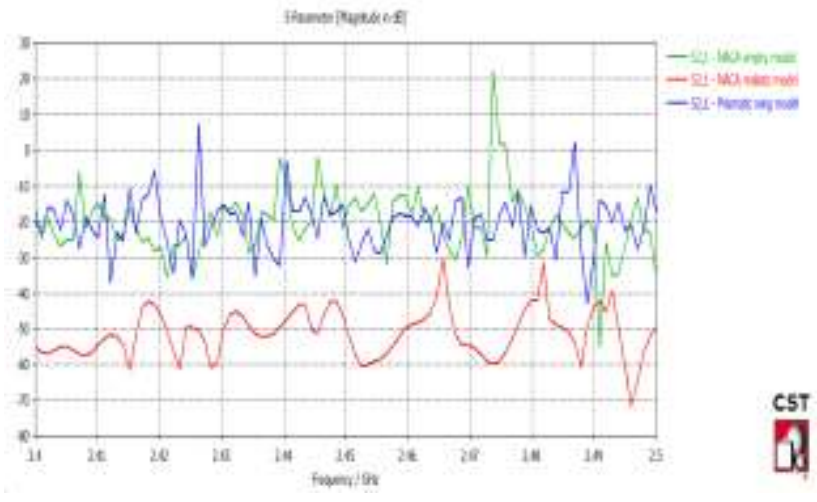

Figure 5. Position: Tip of the wing

The effect of the wing's shape and the inner structure on the S21 parameter value has great impact on the realistic wing model. The parameter value of S21 has decreased for the realistic wing model and for the empty, prismatic wing model S21 parameter value has not undergone drastic changes.

From the analysis of the simulation results, it is evident that the rectangular shape of the wing has main effect on resonance over the radiowave propagation and also over the several reflections present from the rounded shapes. There is no significant difference between the various nodes which between the frequency range of $2.4 \mathrm{GHz}$ and the $2.45 \mathrm{GHz}$. When the frequency level is higher than $2.6 \mathrm{GHz}$, large variations are shown on coupling at the placement of the antenna near $1 / 2$ position of the wing. The presence of this high difference is due to varying coupling present among the different antennas, which have occurred mainly on the higher part of the $2.4 \mathrm{GHz}$ frequency.

Both in the realistic model and the empty wing model, there is an increase in coupling when the frequency is increased in the ZigBee band. From the graphs it is evident that the largest coupling difference of $30 \mathrm{~dB}$ is present when the second receiver is situated at $3 / 4^{\text {th }}$ length of the wing and at the lowest value of coupling difference of $5 \mathrm{~dB}$ is when the placement of antenna is done at half of the wing length. This is mainly because of the difference in the thickness of the wing along the wings length. With respect to the influence of wing inner structure, the highest coupling difference of $30 \mathrm{~dB}$ is present at the frequency of nearly $2.465 \mathrm{GHz}$, when there is large difference between the first and second transceiver. The presence of resonance in the wings inner structure increases the coupling that is present between the nodes when compared to that of the empty wing model. In case of the first ZigBee channel the coupling difference was between $7 \mathrm{db}$ and $15 \mathrm{db}$. This 
is because of the presence of local minimum related to ZigBee band for the complete tested range of frequency.

\section{Conclusion}

In this paper analysis of radiowave propagation in the ZigBee frequency band was carried-out. Three aircraft wing models were considered and designed for the simulations. The simulation result analysis looked at investigating the coupling difference between the antennas with respect to the different antenna position. The estimation of S21 parameter value was made as it helps in assessing the quality of communication link in the environment.

The initial results from this analysis have shown that the received signal level is lower than the minimum ZigBee SNR value that was specified. Hence, these indicate that a higher number of transceivers would be required and this will be part of the ongoing work currently being carried out as part of this project.

Furthermore, as expected the effects of wing shape and wing inner structure had a significant impact on the radiowave propagation inside the wing. With respect to wing shape the difference in the thickness along the wing length results in resonance.

\section{Future work}

As a part of future work, greater number of antennas positions will be considered to identify their influence on radiowave propagation. It is also important to take high complex wing inner structures like pipes and the fuel tank into account. Simulations will also consider different type of mediums that is present inside the aircraft wing i.e. jet fuels.

\section{References}

[1] J. Pinto, G. M. Lewis, J. A. Lord, R. A. Lewis and B. H Wright. Wireless Data Transmission within an Aircraft Environment. Sensor Systems Department. BAE Systems Advanced Technology Centre, Great Baddow, Chelmsford, Essex, CM2 8HN, UK

[2] Gang Xiao, Hainan Diao, Zhongliang Jing. Integrated Aircraft Environment Surveillance System for Large Civil Aircraft. School of Aeronautics and Astronautics, Shanghai Jiaotong University, Shanghai. 2011

[3] Qu Lei, Liu Sheng-de, Hu Xian-bin. ZigBee Technology and Application $[\mathrm{M}]$. Beijing University of Aeronautics and Astronautics Press, 2007.09

[4] Zhan Hao-yan, Sun Chang-song, WU Shan, etc. Application of ZigBee Technology in Coal Mine Rescue System [J]. Computer Engineering and Applications, 2006, 42 (24), p.181-183.

[5] Liu Hui, Wang Mao-hua, Wang Yue-xuan etc. Development of farmland soil moisture and temperature monitoring system based on wireless sensor network [J]. Jilin University (Engineering Science), 2008, 38 (3).p. 604-608.

[6] Zhong Xiao-bo, LI Yan-jun. Research of the Radio Frequency Tag Technology in the Civil Aircraft Maintenance [J]. Aircraft Design, 2011, 31 (1) p.55-60

[7] Satish Kumar Chilakala. Development and Flight Testing of a Wireless Avionics Network Based on the IEEE 802.11 Protocols. ProQuest, 2008

[8] Subhas Chandra Mukhopadhyay, Henry Leung. Advances in Wireless Sensors and Sensor Networks. Springer Science \& Business Media, 16 Apr 2010.

[9] Caojin'Yan zhaowen, Yu wenlu'Shi guochang. Simulation Analysis of Aircraft Electromagnetic Environment. IEEE publications. 2012.

[10] Ismail Hameduddin, Abdulrahman H. Bajodah, "Nonlinear Generalized Dynamic Inversion Aircraft Control," AIAA Guidance, Navigation, and Control Conference, Portland, Oregon, 2011

[11] Whalen, D.; Rathinam, S.; Bagge, C. Advanced developments in airport surface and terminal area traffic surveillance applications. IEEE/AIAA $22^{\text {nd }}$ Digital Avionics Systems Conference, 2003.Page(s): 9.B.3 - 9.1-9 vol.2.

[12] A. Mehta et al., "Design and fabrication of solar R/C model aircraft," IJMER, vol. 3, no. 2, pp. 752-758, 2013.

[13] Alexandru Lavric, Valentin Popa, Codrin Males, Ilie Finis. A Performance Study of ZigBee Wireless Sensors Network Topologies for Street Lighting Control Systems. International Workshop on Mobile Ad-Hoc Wireless Networks. 2012.

[14] Nezih Mrad and Ibrahim Haroun. Wireless sensor networks in air vehicle platforms. Technical report, Communications Research Centre, Defence R\&D Canada - Atlantic, Canada, May 2009.

[15] Justin R. Rufa, Ella M. Atkins. Sensor Fusion for Unmanned Aircraft System Navigation in an Urban Environment. American Control Conference (ACC). Portland, Oregon, USA. June 2014.

[16] Ruben de Francisco, Li Huang, Guido Dolmans, and Harmke de Groot. Coexistence of ZigBee Wireless Sensor Networks and Bluetooth inside a Vehicle. IEEE publications. 2009

[17] Shamneesh Sharma, Keshav kishore and Dinesh Kumar. Wireless Sensor Networks-A Review on topologies and Node Architecture. International Journals of Computer Sciences and Engineering. Vol-1(2), October 2013.

[18] Rinki Sharma, Shreyas K. Gupta, Suhas K.K. and G. Srikanth Kashyap. Performance Analysis of Zigbee based Wireless Sensor Network for Remote Patient Monitoring. Fourth International Conference on Communication Systems and Network Technologies. 2014.

[19] Centeno and N. Alford. Measurement of ZigBee wireless communications in mode-stirred and mode-tuned reverberation chamber. Progress in Electromagnetic Research M, Vol. 18, 171-178, 2011.

[20] Divya Sharma, Sandeep Verma and Kanika Sharma. Network Topologies in Wireless Sensor Networks: A Review. IJECT (International Journals of Electronics and Communication Technology) Vol. 4, Issue Spl - 3, April - June 2013. 